\title{
PRUEBAS DE IDENTIDAD Y PATERNIDAD EN GANADO BRAHMAN REGISTRADO DE COSTA RICA ${ }^{1}$
}

\author{
Jorge Campos ${ }^{*}$, Bernardo Vargas ${ }^{1 / *}$, Jorge Camacho*, Argerie Cruz ${ }^{* *}$ \\ Palabras clave: Microsatélite; probabilidad de exclusión; razón de verosimilitud. \\ Keywords: Microsatellite; exclusion probability; likelihood ratio. \\ Recibido: 03/07/17 \\ Aceptado: 13/09/17
}

RESUMEN

En programas de evaluación genética es indispensable la correcta identificación de los individuos de la población y la certeza de sus relaciones de parentesco. El objetivo de este estudio fue implementar pruebas de identificación y verificación de paternidad mediante 18 marcadores microsatélite en ganado registrado de raza Brahman de Costa Rica. Inicialmente, se calcularon parámetros genéticos poblacionales a partir del análisis de 950 muestras de ADN (7 sementales, 788 vacas y 155 crías) colectadas durante los años 2015/2016, procedentes de 13 hatos de ganado registrado. Los promedios de heterocigosidad observada y esperada fueron de 0,67 y 0,70 , respectivamente. El índice de fijación fue de $4 \%$, con desviaciones significativas del equilibrio Hardy-Weinberg (EHW) en 9 de los 18 loci. Todos los marcadores, excepto ETH225, presentaron contenido de información polimórfica mayor a 0,50 . La probabilidad de identidad combinada con todos los marcadores fue $2,4 \times 10^{-17}$ para individuos seleccionados aleatoriamente y $2,6 \times 10^{-7}$ para hermanos completos. Las probabilidades de exclusión combinada fueron superiores a 0,999 . Todos estos parámetros presentaron valores satisfactorios para la

1 Autor para correspondencia. Correo electrónico: bernardo.vargas.leitón@una.cr

* Universidad Nacional, Posgrado Regional en Ciencias Veterinarias Tropicales, Costa Rica.

\section{ABSTRACT}

Identity and parentage testing in registered Brahman cattle of Costa Rica. In genetic evaluation programs, the correct identification of the individuals of the population and the certainty of their kinship relationships is indispensable. The objective of this study was to implement identity verification and paternity tests using 18 microsatellite markers, in registered Brahman cattle from Costa Rica. Genetic parameters were calculated from the analysis of 950 DNA samples (7 sires, 788 cows and 155 offspring) collected during years 2015/2016, from 13 herds with registered cattle. Average observed and expected heterozygosity were 0.67 and 0.70 , respectively. The fixation index was $4 \%$, with significant deviations of the Hardy Weinberg equilibrium in 9 of the 18 loci. All markers except ETH225 had polymorphic information content greater than 0.50 . The combined probability of identity with all markers was $2.4 \times 10^{-17}$ for randomly selected individuals and $2.6 \times 10^{-7}$ for full siblings. The combined probabilities of exclusion were greater than 0.999. All these parameters presented satisfactory values for the accomplishment of tests of identity and paternity. Next, paternity confirmation tests

\footnotetext{
** Instituto Nacional de Innovación y Transferencia en Tecnología Agropecuaria, Costa Rica.
} 
realización de pruebas de identidad y paternidad. Seguidamente, se realizaron pruebas de confirmación de paternidad en 137 crías con padre y/o madre reportados procedentes de 3 hatos. Los porcentajes de rechazo de paternidad basados en el principio de exclusión (al menos 2 loci discordantes) vs. índices críticos de verosimilitud, fueron respectivamente: $18,2 \%$ vs. $24,1 \%$ para las madres, $14,0 \%$ vs. $12,5 \%$ para los padres, y $34,6 \%$ vs. $26,3 \%$ para los tríos. Se observaron diferencias significativas entre hatos para el porcentaje de rechazo de paternidad, oscilando desde 15,6 a 39,4\% en madres, 0 a $30 \%$ en padres, y 19,5 a $31,8 \%$ en tríos. Se demuestra la necesidad de un mayor control en los apareamientos y en el registro de información genealógica en estos hatos.

\section{INTRODUCCIÓN}

El hato bovino costarricense está compuesto por 1278817 animales, de los cuales un $42,1 \%$ se clasifica como ganado de carne (INEC 2015). En los sistemas de ganado de carne el tipo racial predominante es el cebú y la raza más común es la Brahman (CORFOGA 2012). La raza Brahman predomina en el 39,7\% de las fincas ganaderas del país (CORFOGA 2012).

Debido a la importancia de esta raza, desde hace varios años se creó el Programa Nacional de Evaluación y Mejoramiento Genético de la raza Brahman en Costa Rica, que tiene por objetivo identificar y promover el uso de los animales genéticamente superiores (Cruz Méndez 2015), ya que como etapa previa a la implementación de planes de mejora genética, es importante conocer la integridad de la información genealógica presente en las explotaciones ganaderas (Domínguez et al. 2012, Cruz Méndez 2015).

El análisis de ADN basado en marcadores microsatélite de tipo STR ha constituido el were performed on 137 offspring with reported father and/or mother from 3 herds. Paternity rejection rates based on the exclusion principle (at least 2 discordant loci) vs. critical likelihood values were, respectively: $18.2 \%$ vs. $24.1 \%$ for mothers, $14.0 \%$ vs. $12.5 \%$ for fathers, and $34.6 \%$ vs. $26.3 \%$ for trios. There were significant differences between herds for the percentage of paternity rejection, ranging from 15.6 to $39.4 \%$ in mothers, 0 to $30 \%$ in fathers, and 19.5 to $31.8 \%$ in trios. The need for greater control in mating and recording of genealogical information in these herds is demonstrated. sistema internacional estándar de verificación de identidad y parentesco en diferentes especies, bajo los lineamientos sugeridos por la Sociedad Internacional para la Genética Animal (ISAG 2014). Recientemente los marcadores de tipo SNP son también utilizados con éxito para este fin (ISAG 2014).

Para fines de verificación de identidad, la utilidad de un conjunto de marcadores microsatélite puede medirse mediante la probabilidad de identidad combinada, que se define como la probabilidad de que 2 individuos seleccionados aleatoriamente dentro de una población presenten genotipos idénticos para los loci en cuestión (Waits et al. 2001). Esta probabilidad depende del número de alelos y la distribución de frecuencia de estos alelos en la población bajo estudio (Ichikawa et al. 2001, Curi y Lopes 2002). En pruebas de verificación de identidad, generalmente se requiere que los marcadores utilizados provean en probabilidad de identidad combinada menor que 0,00001 . 
En relación con el análisis de paternidad, se utilizan diferentes enfoques estadísticos (Jones et al. 2010). Entre los 2 enfoques más utilizados en la práctica están el de "principio de exclusión" y el de "índices de verosimilitud".

En el enfoque basado en exclusión los genotipos de los padres candidatos se comparan con el genotipo de la progenie y se tiene en cuenta el genotipo del otro progenitor, si está disponible. Los padres candidatos se excluyen como padres si se producen discordancia en al menos 2 loci (Jones et al. 2010). El poder específico de cada microsatélite para excluir un individuo dentro de un conjunto aleatorio de padres potenciales se mide en términos de su probabilidad de exclusión (Ichikawa et al. 2001). Se requiere que el conjunto de microsatélites utilizado garantice una probabilidad de exclusión combinada mayor de 99,9\%.

En el segundo enfoque para cada padre seleccionado y cada locus, se calcula la razón de verosimilitud (likelihood ratio- LR, Jones et al. 2010), que indica la posibilidad que tiene el individuo de ser el padre o no. El logaritmo natural del producto de las LR obtenidas para todos los loci (Score LOD) es una medida que permite comparar entre varios padres potenciales.

El presente estudio tiene por objetivo implementar el uso de pruebas de ADN por microsatélites con fines de identificación y verificación de paternidad en ganado bovino registrado de raza Brahman en Costa Rica.

\section{MATERIALES Y MÉTODOS}

\section{Procedencia y procesamiento de las muestras}

Para el cálculo de los parámetros genéticos poblacionales, se contó con un total de 950 muestras de pelo de animales Brahman (7 sementales, 788 vacas, 155 crías) registrados en la Asociación de Criadores de Ganado Cebú de Costa Rica. Estas muestras fueron recolectadas durante 2015 y 2016, y procedieron de 13 hatos voluntarios entre los 17 participantes en el Programa Nacional de Evaluación Genética de la raza Brahman (Cruz Méndez 2015).
El material de muestreo consistió en folículos pilosos de la raíz de la cola del bovino, arrancados manualmente mediante un solo movimiento rápido y continuo a contrapelo, se siguieron las recomendaciones de Cordero et al. (2013). Se corroboró que las muestras fueran de pelo seco, libre de excrementos y orina. Estas muestras fueron colocadas en sobres individuales con la información del animal y el hato de procedencia.

La extracción del ADN fue realizada en el Laboratorio de Bioseguridad del LANASEVE del Servicio Nacional de Salud Animal del estado (SENASA); se seleccionaron aproximadamente 30 pelos de la cola con folículo, según el procedimiento descrito por Cordero et al. (2013).

La amplificación de ADN por PCR se realizó utilizando el kit Thermo Scientific ${ }^{\mathrm{TM}}$ Bovine Genotypes Panel 3.1. Este kit incluyó un total de 18 marcadores, 12 de los cuales corresponden a los recomendados por ISAG (FAO 2011) para uso en pruebas de parentesco en bovinos (BM1818, ETH10, TGLA122, BM2113, SPS115, BM1824, INRA023, TGLA126, TGLA53, ETH3, ETH225, TGLA227). El kit incluyó otros 6 marcadores adicionales (CSSM66, ILSTS006, CSRM60, SPS113, MGTG4B y RM067). El cromosoma de localización de cada marcador, el rango del tamaño de los alelos y la secuencia de los cebadores que los delimitan se detallan en Campos (2017).

Los productos de la amplificación fueron sometidos a electroforesis capilar al utilizar un analizador genético ABI 3130, según el procedimiento descrito por Cordero et al. (2013). Los polimorfismos de los microsatélites fueron discriminados de acuerdo con sus patrones de fluorescencia y tamaño. Los datos se visualizaron e identificaron mediante el software GeneMapper v3.0 (Applied Byosistems 2002).

\section{Estimación de parámetros genéticos poblacionales}

Los perfiles genéticos obtenidos en el paso anterior fueron analizados mediante el programa 
Cervus (Marshall et al. 1998, Kalinowski et al. 2007) y se obtuvieron los siguientes parámetros genéticos poblacionales: el número total de alelos por marcador $(\mathrm{Na})$, el número efectivo de alelos $\left(N e=1 / \Sigma p^{2}\right.$, con $p=$ frecuencias alélicas por marcador), la heterocigosidad observada (Ho, obtenida como la proporción de individuos heterocigóticos en la muestra), la heterocigosidad esperada $\left(H e=1-\Sigma_{i}^{n}=1 P_{1}^{2}\right)$; el índice de fijación $\left(F=1-\frac{H o}{H e}\right)$, y el contenido de información polimórfica $\left(C I P=1-\Sigma_{i}^{n}=1 P_{1}^{2}-\right.$ $\left(\Sigma_{i}^{n}=1 \Sigma_{j}^{n}=i=12 p_{i}^{2} p_{j}^{2}\right)$ Además, se evaluó la hipótesis de equilibrio Hardy-Weinberg al utilizar la prueba Chi-cuadrado y se obtuvo un estimado de la frecuencia de alelos nulos (Summers y Amos 1997).

\section{Probabilidades de identidad y exclusión}

Para evaluar la eficiencia del set de marcadores como herramienta para pruebas de verificación de identidad y paternidad en la población Brahman local, se obtuvieron estimados de Probabilidad de Identidad (PI y $\mathrm{PI}_{S I B S}$ ) para cada locus mediante el módulo de identidad del programa Cervus (Marshall et al. 1998, Kalinowski et al. 2007). PI se obtiene a partir de la fórmula según Waits et al. 2001

$$
P I=\sum_{i=1}^{n}\left(p_{i}^{2}\right)^{2}+\sum_{i=1}^{n} \sum_{j=i+1}^{n}\left(2 p_{i} p_{j}\right)^{2}
$$

Se interpreta como la probabilidad de que 2 individuos seleccionados aleatoriamente en la población presenten genotipos idénticos para un locus.

$\mathrm{PI}_{\text {SIBS }}$ se obtiene como:

$$
P I_{\text {SIBS }}=0,25+\left(0,5 \sum_{i=1}^{n} p_{i}^{2}\right)+\left[0,5\left(\sum_{i=1}^{n} p_{i}^{2}\right)^{2}\right]-\left(0,25 \sum_{i=1}^{n} p_{i}^{4}\right)
$$

Se interpreta como la probabilidad de que 2 hermanos completos seleccionados en la población presenten genotipos idénticos para un locus. $\mathrm{PI}_{S I B S}$ es un estimado más conservador recomendado en poblaciones consanguíneas o con desviaciones marcadas del equilibrio Hardy Weinberg.

La Probabilidad de Identidad Combinada (PIC) para el set de marcadores se calculó como el producto de las PI específicas de cada marcador.

Seguidamente, se calculó la probabilidad de exclusión (Jamieson y Taylor 1997), definida como la probabilidad de que un individuo seleccionado aleatoriamente de la población sea excluido como potencial padre de una cría. Esta probabilidad se obtuvo para los siguientes 3 casos de evaluación de paternidad:

a) $\mathrm{PE}_{1 \mathrm{P}}$ : Un padre candidato, otro padre conocido, calculado como

$$
P E_{1 P}=1-2 \Sigma p_{i}^{2}+\Sigma p_{i}^{3}+2 \Sigma p_{i}^{4}-3 \sum p_{i}^{5}-2\left(\sum p_{i}^{2}\right)^{2}+3 \Sigma p_{i}^{2} \Sigma p_{i}^{3},
$$

b) $\mathrm{PE}_{2 \mathrm{P}}$ : Un padre candidato, otro padre desconocido, obtenido como:

$$
P E_{2 P}=1-4 \sum p_{i}^{2}+2\left(\sum p_{i}^{2}\right)^{2}+4 \sum p_{i}^{3}-3 \sum p_{i}^{4},
$$

c) $\mathrm{PE}_{\mathrm{PP}}$ : Par de padres candidatos:

$$
P E_{P P}=1+4 \Sigma p_{i}^{4}+4 \Sigma p_{i}^{5}-3 \sum p_{i}^{6}-8\left(\sum p_{i}^{2}\right)^{2}+8\left(\sum p_{i}^{2}\right)\left(\sum p_{i}^{3}\right)+2\left(\Sigma p_{i}^{3}\right)^{2} \text {. }
$$

El cálculo de Probabilidades de Exclusión Combinadas (PEC) para el set completo de marcadores se realizó mediante la fórmula:

$$
P E C=1-\prod_{i=1}^{n}\left(1-P E_{i}\right) .
$$




\section{Verificación de paternidad en 3 hatos}

Una vez confirmada la eficiencia del set de marcadores, se realizó una evaluación de la integridad de las paternidades reportadas en 3 de los hatos, según anuencia de sus propietarios, para lo cual se contó con los perfiles genéticos de 155 crías, entre las cuales 118 contaron con el perfil genético de ambos padres, 19 contaron solo con el perfil genético de sus madres y los 18 restantes contaron solo con el perfil de genético de sus padres. Para estos casos, los perfiles genéticos fueron comparados mediante el módulo Par de Padres con Sexo Conocido del programa Cervus (Marshall et al. 1998, Kalinowski et al.2007) y se contabilizaron los casos de discordancias de genotipo entre progenie vs. padres candidatos.

Para cada padre candidato y cada locus se obtuvo un estimado de la razón de verosimilitud LR $=x / p$, donde $x$ es la probabilidad de transmisión del alelo obligado (1 para homocigotas y 0,5 para heterocigotas) y $p$ es la frecuencia de la variante alélica respectiva. La existencia de padres conocidos permitió la identificación de los alelos obligados. LR se interpreta cómo cuántas veces más (o menos) posibilidad tiene el padre candidato de ser el verdadero padre de la cría que de no serlo. LR se ajustó, además, por la tasa de error de genotipado, estimada a partir del análisis de discordancias padres vs. progenie.

Finalmente, se calculó el Score LOD, calculado como el logaritmo natural del producto de las LR de todos los loci. Este score permite discriminar entre varios padres potenciales, aun en presencia de errores de genotipado. Los Score LOD fueron cotejados contra distribuciones de probabilidad obtenidas a partir de simulación por remuestreo (Boostrapping), con el fin de determinar el nivel de confianza logrado en la asignación de las paternidades. Se asumieron parámetros de simulación en función de las características de la población analizada, entre ellos: tasa de error de genotipado: $7 \%$, madres candidatas: 118 , padres candidatos: 7 , porcentaje de padres y madres genotipados: $75 \%$, parentesco entre padres verdaderos: $3 \%$, proporción de la población consanguínea: $10 \%$. El tamaño de progenie simulada fue de 100000 individuos, según lo sugerido por Marshall et al. (1998) y Kalinowski et al. (2007). Se obtuvieron los valores críticos de LOD para rechazo de paternidad con niveles de 95 y $99 \%$ de confianza y se compararon los porcentajes de rechazo de paternidad de los 3 hatos mediante prueba de chi-cuadrado y comparaciones pareadas de proporciones.

\section{RESULTADOS Y DISCUSIÓN}

\section{Parámetros de diversidad alélica}

El promedio de alelos observados por marcador fue de 11,5 (Cuadro 1), el cual oscila entre 6 (BM1824, ETH10) y 17 (SPS113). Muchos alelos se presentaron en frecuencias muy bajas $(<1 \%)$, por lo que el promedio efectivo de alelos por marcador fue de solo 3,76. El número de alelos observados es menor al reportado por estudios similares, por ejemplo 14,2 (Hernández et al. 2009), 13,9 (Gómez et al. 2013) o 12 (Martínez et al. 2015). Esto se debe probablemente a los mayores tamaños de muestra utilizados en dichos estudios, lo que permitió identificar alelos suplementarios, y también, a la mayor heterogeneidad racial de dichas poblaciones, ya que incluyeron animales no registrados de poblaciones comerciales. 
Cuadro 1. Parámetros de diversidad alélica $(\mathrm{Na}, \mathrm{Ho}, \mathrm{He}, \mathrm{F}, \mathrm{CIP})^{1}$, frecuencia estimada de alelos nulos (f-Nul), valor de significancia de la prueba Hardy-Weinberg ${ }^{2}$, probabilidades de identidad $\left(\mathrm{PI}_{\mathrm{PI}} \mathrm{SIBS}\right)^{3}$ y probabilidades de exclusión $\left(\mathrm{PE}_{1 \mathrm{P}}\right.$, $\mathrm{PE}_{2 \mathrm{P}}$ y $\left.\mathrm{PE}_{\mathrm{PP}}\right)^{4}$ para cada uno de los 18 marcadores microsatélite en una muestra de 950 individuos de la población de ganado Brahman registrado de Costa Rica.

\begin{tabular}{lrrrrrrrrrrrr}
\hline Locus & $\mathrm{Na}$ & $\mathrm{Ho}$ & $\mathrm{He}$ & $\mathrm{F}$ & $\mathrm{CIP}$ & $\mathrm{f}-\mathrm{Nul}$ & $\mathrm{HW}$ & $\mathrm{PI}$ & $\mathrm{PI}_{\mathrm{SIBS}}$ & $\mathrm{PE}_{1 \mathrm{P}}$ & $\mathrm{PE}_{2 \mathrm{P}}$ & $\mathrm{PE}_{\mathrm{PP}}$ \\
\hline BM1818 & 11 & 0,68 & 0,77 & 0,13 & 0,74 & 0,07 & $* * *$ & 0,08 & 0,38 & 0,57 & 0,39 & 0,76 \\
BM1824 & 6 & 0,58 & 0,61 & 0,04 & 0,53 & 0,02 & $\mathrm{NS}$ & 0,23 & 0,51 & 0,33 & 0,19 & 0,49 \\
BM2113 & 12 & 0,74 & 0,75 & 0,01 & 0,72 & 0,00 & $*$ & 0,09 & 0,40 & 0,56 & 0,37 & 0,76 \\
CSRM60 & 13 & 0,65 & 0,69 & 0,06 & 0,67 & 0,03 & $* * *$ & 0,12 & 0,43 & 0,49 & 0,30 & 0,70 \\
CSSM66 & 9 & 0,71 & 0,74 & 0,04 & 0,71 & 0,02 & $\mathrm{NS}$ & 0,10 & 0,40 & 0,53 & 0,35 & 0,72 \\
ETH10 & 6 & 0,65 & 0,66 & 0,02 & 0,60 & 0,01 & $\mathrm{NS}$ & 0,17 & 0,46 & 0,40 & 0,24 & 0,58 \\
ETH225 & 11 & 0,46 & 0,48 & 0,03 & 0,44 & 0,01 & $* * *$ & 0,31 & 0,59 & 0,27 & 0,12 & 0,43 \\
ETH3 & 12 & 0,59 & 0,62 & 0,05 & 0,54 & 0,03 & $\mathrm{NS}$ & 0,22 & 0,50 & 0,35 & 0,21 & 0,51 \\
ILSTS006 & 14 & 0,55 & 0,65 & 0,16 & 0,60 & 0,09 & $* * *$ & 0,17 & 0,47 & 0,41 & 0,25 & 0,59 \\
INRA23 & 11 & 0,80 & 0,78 & $-0,02$ & 0,75 & $-0,01$ & $\mathrm{NS}$ & 0,08 & 0,38 & 0,59 & 0,41 & 0,79 \\
MGTG4B & 11 & 0,77 & 0,78 & 0,00 & 0,75 & 0,00 & $\mathrm{NS}$ & 0,08 & 0,38 & 0,58 & 0,40 & 0,78 \\
RM067 & 8 & 0,51 & 0,57 & 0,10 & 0,53 & 0,05 & $* * *$ & 0,23 & 0,52 & 0,35 & 0,18 & 0,53 \\
SPS113 & 17 & 0,68 & 0,71 & 0,04 & 0,67 & 0,02 & $* *$ & 0,12 & 0,43 & 0,49 & 0,31 & 0,69 \\
SPS115 & 13 & 0,76 & 0,74 & $-0,02$ & 0,70 & $-0,01$ & $\mathrm{NS}$ & 0,11 & 0,41 & 0,52 & 0,34 & 0,71 \\
TGLA122 & 16 & 0,89 & 0,89 & 0,00 & 0,88 & 0,00 & $\mathrm{NS}$ & 0,02 & 0,31 & 0,77 & 0,63 & 0,92 \\
TGLA126 & 12 & 0,79 & 0,82 & 0,04 & 0,80 & 0,02 & $*$ & 0,05 & 0,35 & 0,65 & 0,48 & 0,83 \\
TGLA227 & 9 & 0,62 & 0,65 & 0,04 & 0,59 & 0,02 & NS & 0,18 & 0,47 & 0,39 & 0,23 & 0,57 \\
TGLA53 & 16 & 0,64 & 0,69 & 0,08 & 0,65 & 0,04 & $* * *$ & 0,14 & 0,44 & 0,46 & 0,29 & 0,65 \\
Promedio & 11,5 & 0,67 & 0,70 & 0,04 & 0,66 & 0,02 & & & & & & \\
Combinada & & & & & & & & 2,4 & 2,6 & $>$ & $>$ & $>$ \\
& & & & & & & & $\times 10^{-17}$ & $\times 10^{-7}$ & 0,999 & 0,999 & 0,999 \\
\hline
\end{tabular}

$1 \mathrm{Na}=$ Número total de alelos, Ho= Heterocigosis observada, He= Heterocigosis esperada, $\mathrm{F}=$ Índice de fijación, $\mathrm{CIP}=$ contenido de Información Polimórfica.

2 Significancia estadística: NS=no significativo, * $\mathrm{p}<0,05, * * \mathrm{p}<0,01, * * * \mathrm{p}<0,001$.

3 PI= Probabilidad de Identidad entre individuos seleccionados aleatoriamente, $\mathrm{PI}_{\text {SIBS }}=$ Probabilidad de Identidad entre hermanos completos (no gemelos)

$4 \quad \mathrm{PE}_{1 \mathrm{P}}=$ Probabilidad de exclusión de paternidad, un padre candidato y otro padre conocido, $\mathrm{PE}_{2 \mathrm{P}}=$ Probabilidad de exclusión de paternidad, un padre candidato y otro padre desconocido, $\mathrm{PE}_{\mathrm{PP}}=$ Probabilidad de exclusión de paternidad, padre y madre candidatos.

La heterosis observada fue menor que la esperada para casi todos los marcadores, con excepción de INRA23 y SPS115 (Cuadro 1). Los promedios de heterosis observada y esperada fueron de 0,67 y 0,70, respectivamente. Las desviaciones (Ho-He) oscilaron entre 0 (TGLA122) y 0,13 (TGLA126). El índice de fijación (F) osciló entre -0,02 (INRA23 y SPS115) y 0,16 
(ILSTS006), con un promedio 0,04. Esto sugiere una tendencia a la consanguinidad en la población analizada, lo cual es de esperar en una población de animales registrados altamente emparentados, sometidos a selección y con apareamientos dirigidos (Cervini et al. 2006, Gómez et al. 2013). Es probable que estas mismas razones causaran que la prueba de equilibrio Hardy-Weinberg fuera significativa para 9 de los 18 marcadores (Cuadro 1).

Todos los marcadores utilizados estuvieron en la categoría de altamente informativos (CIP $>0,5)$, a excepción del marcador ETH225 $(\mathrm{CIP}=0,44)$, considerado medianamente informativo (Cuadro 1). Los demás marcadores se encontraron en el rango de CIP entre 0,53 (BM1824) y 0,88 (TGLA122), con un promedio de 0,66. Estudios previos en animales de registro reportaron promedios de CIP muy similares, tales como 0,64 (Cervini et al. 2006), 0,66 (Hernández et al. 2009) o 0,65 (Gómez et al. 2013). Otros estudios en poblaciones comerciales reportaron valores superiores, tales como 0,70 (Carolino et al. 2009), 0,72 (Stevanovic et al. 2010) o 0,75 (Martínez et al. 2015). Esto puede ir ligado a la mayor heterogeneidad racial presente en dichas poblaciones.

La frecuencia promedio de alelos nulos fue de 0,02 (Cuadro 1), con los valores más altos para BM1818 (7\%) y ILSTS006 (9\%). De acuerdo con Cervini et al. (2006), altas frecuencias $(>5 \%)$ de alelos nulos pueden causar también una deficiencia de heterocigosidad y desviaciones aparentes del equilibrio de Hardy-Weinberg en los loci. Asimismo, en las pruebas de parentesco pueden dar origen a falsos negativos en la asignación de padres y madres.

\section{Probabilidades de identidad y exclusión}

Mediante el uso combinado de los 18 marcadores se obtuvieron valores de PIC de $2,4 \times 10^{17}$ y $2,6 \times 10^{-7}$ (Cuadro 1). Los loci con mayor poder discriminatorio, o sea menor PI, fueron TGLA122 y TGL126, que a su vez fueron los que presentaron mayor heterocigosidad y CIP. Por el contrario, el locus de menor poder discriminatorio fue ETH225, para el cual existió un alelo altamente predominante con frecuencia de 0,70 Es importante señalar que la informatividad de un marcador puede variar en función de la raza. En un estudio previo a nivel local, que incluyó diversos tipos raciales (Cordero et al. 2015), el marcador ETH225 mostró una distribución más homogénea de sus alelos, con un valor de 0,29 para el alelo más frecuente y un valor de CIP de 0,79 , muy superior al 0,44 obtenido en el presente estudio.

En cuanto a las probabilidades de exclusión, los resultados indicaron que los loci con mayor poder de exclusión fueron nuevamente TGLA122 y TGL126, y el de menor poder el ETH225 (Cuadro 1). Las probabilidades de exclusión combinadas fueron en todos los casos mayores a 0,999 , por lo que son consideradas satisfactorias para pruebas de paternidad.

En un estudio previo a nivel local se reportó una PEC de 0,999 con el uso de 18 marcadores en animales comerciales de diferentes características raciales (Cordero et al. 2013). Otros estudios similares en la raza Brahman reportan valores semejantes, tales como 0,999 (Riojas et al. 2009) y 0,993 (Gómez et al. 2013), con el uso de 11 y 8 marcadores, respectivamente. Algunos estudios en otras razas reportaron valores de PEC que variaron de 0,842 a 0,999 al utilizar entre 6 a 10 marcadores microsatélite (Baron et al. 2002, Curi y Lopes 2002, Visscher et al. 2002, Carolino et al. 2009). En todos los estudios se reitera la importancia del uso de los marcadores con mayor poder discriminatorio, ya que al descartar marcadores de baja informatividad, se puede aumentar la PEC con un menor número de marcadores. En el caso específico del presente estudio, el marcador ETH225 fue de poca utilidad por su bajo poder discriminatorio.

\section{Análisis de discordancias por locus}

En el análisis de discordancias por locus se observó que un 66\% (38/58) y un 50\% (24/48), de los genotipos discordantes en madres y padres reportados, respectivamente, podrían ser atribuidos a alelos nulos (Cuadro 2). La tasa promedio de error de genotipado fue alta (10,8 y $9 \%)$, 
no obstante, es probable que este sea un valor sobreestimado, ya que en su cálculo se asume que los padres conocidos han sido identificados con $100 \%$ de certeza (Marshall et al. 1998, Kalinowski et al. 2007). Algunos loci presentaron estimados de tasas de error de genotipado $\geq 15 \%$, tales como: BM1818, ETH225, ETH3, TGLA227, y TGLA53. Las variables tasas de error de genotipado entre loci sugieren que puede haber un subconjunto de loci que no se puede genotipar con fiabilidad, o que pueden tener alelos nulos segregándose en frecuencias moderadas o altas (Marshall et al. 1998, Kalinowski et al. 2007). En estos casos, una alternativa es omitir del análisis de parentesco estos loci (Marshall et al. 1998, Kalinowski et al. 2007).

Cuadro 2. Número de discordancias entre genotipos de padres conocidos vs. progenie, con estimado de alelos nulos y porcentaje de error de genotipado, en función de cada locus.

\begin{tabular}{lcccccc}
\hline \multirow{2}{*}{ Locus } & \multicolumn{2}{c}{ Madre conocida vs. progenie $(\mathrm{n}=137)$} & \multicolumn{2}{c}{ Padre conocido vs. progenie $(\mathrm{n}=136)$} \\
\cline { 2 - 7 } & $\begin{array}{c}\text { Casos } \\
\text { discordantes }\end{array}$ & $\begin{array}{c}\text { Alelos } \\
\text { Nulos }^{1}\end{array}$ & $\begin{array}{c}\text { \% Error de } \\
\text { Genotipado }\end{array}$ & $\begin{array}{c}\text { Casos } \\
\text { discordantes }\end{array}$ & $\begin{array}{c}\text { Alelos } \\
\text { Nulos }\end{array}$ & $\begin{array}{c}\% \text { Error de } \\
\text { Genotipado }\end{array}$ \\
\hline BM1818 & 22 & 12 & $20,5 \%$ & 8 & 3 & $7,5 \%$ \\
BM1824 & 3 & 0 & $5,6 \%$ & 0 & 0 & $0,0 \%$ \\
BM2113 & 9 & 2 & $8,9 \%$ & 11 & 0 & $10,9 \%$ \\
CSRM60 & 5 & 1 & $6,0 \%$ & 7 & 2 & $8,5 \%$ \\
CSSM66 & 9 & 0 & $9,4 \%$ & 6 & 0 & $6,3 \%$ \\
ETH10 & 5 & 1 & $7,6 \%$ & 8 & 1 & $12,3 \%$ \\
ETH225 & 5 & 2 & $15,1 \%$ & 9 & 7 & $27,4 \%$ \\
ETH3 & 10 & 1 & $17,7 \%$ & 4 & 1 & $7,1 \%$ \\
ILSTS006 & 8 & 4 & $11,9 \%$ & 3 & 0 & $4,5 \%$ \\
INRA23 & 9 & 0 & $8,0 \%$ & 5 & 1 & $4,5 \%$ \\
MGTG4B & 11 & 1 & $10,0 \%$ & 5 & 0 & $4,6 \%$ \\
RM067 & 7 & 4 & $14,3 \%$ & 14 & 9 & $28,8 \%$ \\
SPS113 & 7 & 2 & $8,2 \%$ & 10 & 0 & $11,8 \%$ \\
SPS115 & 7 & 0 & $7,5 \%$ & 3 & 0 & $3,2 \%$ \\
TGLA122 & 9 & 0 & $5,2 \%$ & 9 & 0 & $5,2 \%$ \\
TGLA126 & 10 & 0 & $7,6 \%$ & 10 & 0 & $7,7 \%$ \\
TGLA227 & 10 & 5 & $15,9 \%$ & 3 & 0 & $4,8 \%$ \\
TGLA53 & 12 & 3 & $15,0 \%$ & 5 & 0 & $6,3 \%$ \\
Sumatoria & 158 & 38 & & 120 & 24 & $9,0 \%$ \\
Promedio & & & $10,8 \%$ & & & \\
\hline
\end{tabular}

1 Alelos Nulos: estimado a partir de las discordancias de genotipo entre padres y progenie, en las cuales ambos son homocigotas para diferentes alelos, lo que sugiere la existencia de un alelo nulo.

2 Tasa de Error de Genotipado: razón de discordancias en el locus respectivo dividido entre el número total de discordancias, ajustado por la probabilidad de exclusión del locus respectivo ( $\mathrm{PE}_{2 \mathrm{P}}$ en Cuadro 3). 
Las tasas de error para ambos casos, padre o madre conocida, fueron distintas para algunos loci (BM1818, ETH225, ETH3, MGTG4B, RM067, TGLA227). Esto podría deberse a la gran diferencia entre el número de padres $(n=7)$ y madres $(n=137)$ incluidos en el presente estudio, así como a la gran heterogeneidad en las distribuciones alélicas de distintos loci. Por otra parte, la estimación de la tasa de error de genotipado puede verse sesgada por falta de independencia (Marshall et al. 1998, Kalinowski et al. 2007). Una causa obvia de no independencia ocurre cuando existe descendencia múltiple de un mismo padre, hecho que sucedió en el presente estudio con los sementales.

\section{Análisis de paternidad por exclusión}

El análisis de paternidad por exclusión permitió identificar un 58\% de perfiles perfectamente compatibles en el caso de madre vs. progenie y $65 \%$ en el caso de padre vs. progenie (Cuadro 3). En el caso de tríos (padre vs. madre vs. progenie) solo un 35\% de los casos presentó compatibilidad perfecta. Es importante notar que la mayoría de discordancias involucraron solo 1 o 2 loci. Se aprecia nuevamente que un gran número de estas discordancias podrían ser atribuidas a alelos nulos. Los estudios de exclusión requieren al menos 2 loci discordantes entre el candidato y la descendencia para dar cuenta de los errores de tipificación o mutaciones (Jones et al. 2010). En presencia de altas tasas de error y alelos nulos, los casos de una o 2 discordancias probablemente son casos de paternidades correctas, donde las discordancias son causadas por los factores mencionados. Por el contrario, los casos con 3 o más discordancias es más probable que se deban a paternidades incorrectamente reportadas o errores en la identificación de las muestras, ya sea a nivel de campo o en el proceso de laboratorio (Marshall et al. 1998, Kalinowski et al. 2007). 


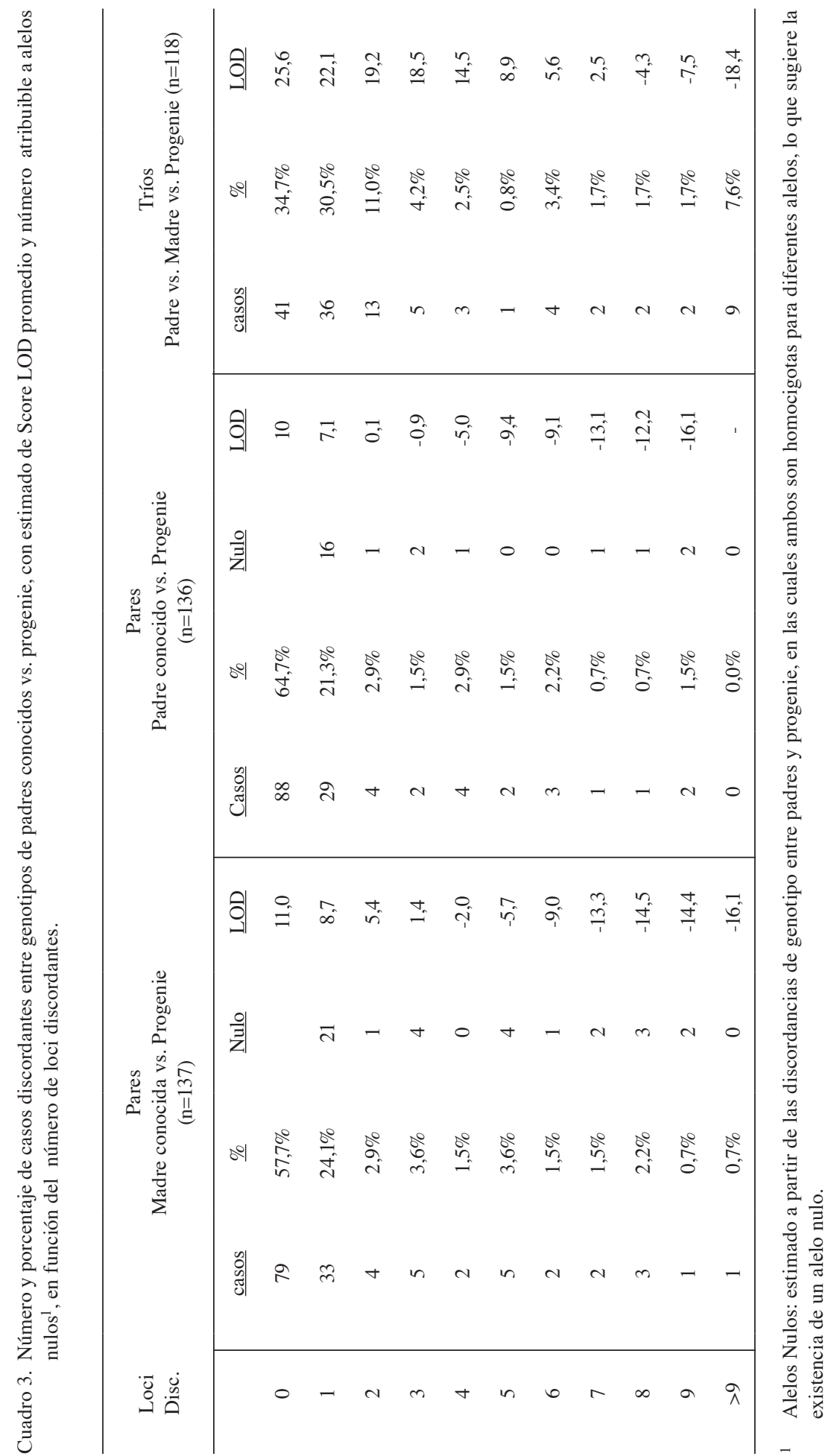


Al aplicar el criterio de exclusión basado en al menos 2 loci discordantes (Jones et al. 2010), los porcentajes de rechazo de paternidad en el presente estudio fueron altos, con valores de $18,2 \%$ en las madres, $14,0 \%$ en los padres y $34,6 \%$ en los tríos. Los resultados reportados por estudios similares son variables. Carolino et al. (2009) reportaron paternidades erróneas, con discordancia en 2 o más loci, de $14 \%$ en madres y $7 \%$ en padres. Se han reportado tasas más altas de paternidades erróneas en ganado Gyr de Brasil (Curi y Lopes 2002), con 27,5\% de rechazo en las paternidades (padre vs progenie vs. madre). Baron et al. (2002), reportaron una tasa de identificación errónea (padre vs. progenie) de $36 \%$ y $22 \%$ en al menos 1 o 2 marcadores, respectivamente. Por otra parte, Rehout et al. (2006) reportaron porcentajes de paternidad errónea en ganado Holstein de 10,7\%.

Existen varios factores que pueden causar discordancia entre perfiles genéticos de padres y progenie, entre los que se mencionan: el registro inadecuado de los animales al momento de la parición, la identificación incorrecta del semen, mal control visual de montas o inseminación artificial y la mala asignación de padres por uso de múltiples toros en grupos de cría (Baron et al. 2002, Carolino et al. 2009).

\section{Análisis de paternidad por índices de verosimilitud}

En el análisis basado en índices de verosimilitud se observó que los promedios de score
LOD disminuyeron conforme aumentó el número de loci discordantes, hasta tomar valores negativos (Cuadro 3). Un valor negativo significa que la probabilidad de paternidad incorrecta es mayor que la de paternidad correcta. En el caso de los padres 3 discordancias fueron suficientes para producir LOD negativos, mientras que en las madres el número requerido fue de $4 \mathrm{y}$ en los tríos se incrementó hasta 8. Esto refleja que el principio de exclusión basado en al menos 2 loci discordantes puede ser insuficiente en algunos casos de discriminación de paternidad.

La evaluación de paternidades mediante valores críticos de LOD mostró que, aun con un nivel de confianza de $95 \%$, existió un alto porcentaje de paternidades rechazadas (Cuadro 4), es decir, probablemente incorrectas. El porcentaje de paternidades rechazadas en madres $(24,1 \%)$ prácticamente duplicó el de los padres $(12,5 \%)$. En el caso de las madres, el criterio de rechazo de paternidad basado en valores críticos de LOD (24,1\% de rechazo, confianza 95\%) resultó ser más estricto que el basado en el criterio de exclusión (18,2\% de rechazo, $\geq 2$ discordancias). Se detectaron 10 casos de incongruencias entre ambos métodos, de los cuales 9 correspondieron a situaciones donde se aceptó la maternidad cuando se utilizó el criterio de exclusión, mientras que se rechazó al utilizar el valor crítico de LOD (95\%). Estas diferencias se deben a que el principio de exclusión no toma en cuenta las frecuencias de los alelos discordantes, los errores de genotipado o la probabilidad de transmisión de los alelos obligados.

Cuadro 4. Número de paternidades asignadas con $99 \%$ y $95 \%$ de confianza y rechazadas con $95 \%$ confianza con base en valores críticos de score de verosimilitud (LOD).

\begin{tabular}{|c|c|c|c|c|c|}
\hline \multirow[t]{2}{*}{ Candidato } & \multicolumn{2}{|c|}{$\begin{array}{l}\text { Paternidad asignada } \\
\text { (99\% Confianza) }\end{array}$} & \multicolumn{2}{|c|}{$\begin{array}{l}\text { Paternidad asignada } \\
\text { (95\% confianza) }\end{array}$} & \multirow{2}{*}{$\begin{array}{c}\begin{array}{c}\text { Paternidad } \\
\text { Rechazada } \\
\text { (95\% Confianza) }\end{array} \\
\mathrm{N}(\%)\end{array}$} \\
\hline & Casos & LOD Crítico & Casos & LOD Crítico & \\
\hline Madre $(n=137)$ & 98 & 7,12 & $98+6$ & 5,42 & $33(24,1 \%)$ \\
\hline Padre $(n=136)$ & 110 & 4,20 & $110+9$ & 1,22 & $17(12,5 \%)$ \\
\hline Tríos (n=118) & 78 & 18,67 & $78+9$ & 16,13 & $31(26,3 \%)$ \\
\hline
\end{tabular}


En el caso de los padres ambos criterios produjeron resultados muy similares (14\% vs. $12,5 \%$ de rechazo), con solo 2 casos de resultados incongruentes, ambos rechazados por el principio de exclusión y aceptados por el criterio LOD. En los tríos se observó que el criterio basado en exclusión fue más estricto $(34,6 \%$ rechazo) que el basado en valores críticos de LOD (26,3\% rechazo, 95\% confianza). Se identificaron 18 casos incongruentes, de los cuales 14 se rechazaron mediante el criterio de exclusión, mientras que se aceptaron al utilizar el valor crítico de LOD (95\%).

El análisis de paternidades rechazadas por hato, basado en valores críticos LOD, demostró que el hato 1 presentó menores porcentajes de rechazo (Cuadro 5). La mayor proporción de rechazos en los casos madre vs. progenie se dieron en el hato 3 , mientras que en los casos padre vs. progenie el mayor problema se presentó en el hato 2. Para los tríos las diferencias no fueron significativas, pero la tasa de rechazo tendió a ser mayor en los hatos 2 y 3 . En el hato 2 la proporción de casos rechazados es alta tanto en madres como padres, mientras que en los hatos 1 y 3 el problema solo se observa en las madres. Un estudio similar en 4 hatos de ganado Boran (Kios et al. 2012) reportó bajas tasas de rechazo, entre 0 y 5\%, para los casos madre vs. progenie, mientras que en los casos padre vs. progenie las tasas de rechazo variaron desde 4,3 hasta $80 \%$.

Cuadro 5. Número de paternidades reportadas (N Rep), rechazadas (N Rec) y porcentaje de rechazo (\% Rec) según hato de procedencia de las muestras.

\begin{tabular}{|c|c|c|c|c|c|c|c|c|c|}
\hline \multirow[t]{2}{*}{ Hato } & \multicolumn{3}{|c|}{$\begin{array}{c}\text { Pares } \\
\text { Madre vs. Progenie }\end{array}$} & \multicolumn{3}{|c|}{$\begin{array}{c}\text { Pares } \\
\text { Padre vs. Progenie }\end{array}$} & \multicolumn{3}{|c|}{$\begin{array}{c}\text { Tríos } \\
\text { Padre vs. Madre vs. Progenie }\end{array}$} \\
\hline & N Rep. & N Rec. & $\% \operatorname{Rec}^{1}$ & N Rec. & N Rec. & $\% \operatorname{Rec}^{1}$ & N Rep. & N Rec. & $\% \operatorname{Rec}^{1}$ \\
\hline 1 & 45 & 7 & $15,6 \% \mathrm{a}$ & 46 & 0 & $0,0 \% \mathrm{a}$ & 41 & 8 & $19,5 \% \mathrm{a}$ \\
\hline 2 & 59 & 13 & $22,0 \% \mathrm{ab}$ & 50 & 15 & $30,0 \% \mathrm{~b}$ & 44 & 14 & $31,8 \% \mathrm{a}$ \\
\hline 3 & 33 & 13 & $39,4 \% \mathrm{~b}$ & 40 & 2 & $5,0 \% \mathrm{a}$ & 33 & 9 & $27,3 \% \mathrm{a}$ \\
\hline
\end{tabular}

1 Porcentajes de rechazo de distintos hatos que comparten una misma letra no difieren significativamente $(\mathrm{p}>0,05)$.

En el presente estudio, las diferencias observadas entre hatos pueden estar ligadas a distintos niveles de eficiencia y rigurosidad tanto en el control de los apareamientos como en la toma de los registros. En el caso del hato 1, se comprobó que existe un mayor nivel de supervisión, lo que es consistente con los menores porcentajes de paternidades rechazadas. En el hato 2 se comprobó que el nivel de supervisión es menor, el manejo del hato es más extensivo y se utiliza la monta natural en la mayoría de los apareamientos. Esto puede explicar los altos porcentajes de rechazo en padres vs. progenie, probablemente asociados a la falta de control al reportar los padres o al momento de las montas.
Por último, se comprobó que el hato 3, aunque cuenta con profesionales a cargo, tiene una menor supervisión y orden en el manejo de los registros. Además, en este hato existe un lote de vacas que presentan problemas para la obtención de pedigríes de sus crías por falta de actualización de la información en la asociación.

En conclusión, los resultados obtenidos reflejan que el set de marcadores utilizado provee una capacidad discriminatoria satisfactoria para fines de verificación de identidad y parentesco. La verificación de paternidad mediante criterios de exclusión basados en un mínimo de 2 loci discordantes puede ser insuficiente en algunos casos, ya que no considera la frecuencia de los 
alelos implicados en la población. En este sentido, los índices de verosimilitud tienen mayor poder discriminatorio, puesto que consideran las frecuencias alélicas y las probabilidades de transmisión, al ser más útiles sobre todo cuando se trata de excluir entre varios padres potenciales. Los resultados hacen ver, además, la necesidad de implementar en los hatos mejores mecanismos de control en los apareamientos y en la toma de registros genealógicos.

\section{LITERATURA CITADA}

Applied Biosystems. 2002. ABI PRISM ${ }^{\circledR}$ GeneMapper ${ }^{\mathrm{TM}}$ Software Versión 3.0 User's Manual. 342 p. (en línea, programa informático). Consultado 24 oct. 2015. Disponible en http://www3.appliedbiosystems. com/cms/groups/mcb_support/documents/ generaldocuments/cms_041304.pdf

Baron,EE; Martínez, ML; Verneque, RS; Coutinho,LL. 2002. Parentage testing and effect of misidentification on the estimation of breeding value in Gyr cattle. Genetics and Molecular Biology 25(4):389-394.

Campos, J. 2017. Uso de marcadores genéticos para análisis de diversidad genética y pruebas de verificación de identidad y paternidad en bovinos Brahman registrados de Costa Rica. Tesis M.Sc., Heredia, Costa Rica, Posgrado Regional en Ciencias Veterinarias Tropical, Universidad Nacional. 81 p.

Carolino, I; Sousa, CO; Ferreira, S; Carolino, N; Silva, FS; Gama, LT. 2009. Implementation of a parentage control system in Portuguese beef-cattle with a panel of microsatellite markers. Genetics and Molecular Biology 32(2):306-311.

Cervini, M; Henrique, F; Mortari, N; Matheucci, E. 2006. Genetic variability of 10 microsatellite markers in the characterization of Brazilian Nellore cattle (Bos indicus). Genetics and Molecular Biology 29(3):486-490.

Cordero, JM; Rojas, G; Vargas, B; León, B. 2013. Uso de marcadores moleculares para identificación y verificación de parentescos en ganado bovino. Informe Final. Proyecto FI-370-11 CONICIT. 16 p.

Cordero, JM; Vargas, B; León, B; Chacón, I; Martínez, M. 2015. Diversidad genética en bovinos de ocho regiones en Costa Rica. Agronomía Mesoamericana 26(2):191-202.

CORFOGA (Corporación Ganadera). 2012. Informe encuesta ganadera-2012 (en línea). 72 p. Consultado 23 set. 2015. Disponible en http://www.corfoga.org/ estadisticas/poblacion-animal/

Cruz Méndez, A. 2015. Evaluación de la variabilidad genética de la raza Brahman en Costa Rica (en línea). 19 p. Consultado 30 set. 2016. Disponible en http://www. corfoga.org/mejoramiento-genetico/
Curi, RA; Lopes, CR. 2002. Evaluation of nine microsatellite loci and misidentification paternity frequency in a population of Gyr breed bovines. Brazilian Journal of Veterinary Research and Animal Science 39(3):129-135.

Domínguez, J; Rodríguez, F; Ortega, J; Santellano, E. 2012. Análisis de la Información genealógica y estimación de parámetros de poblaciones en bovinos de lidia y equinos de pura raza española de México. Revista Científica 22(5):443-450.

FAO (Organización de las Naciones Unidas para la Agricultura y la Alimentación). 2011. Molecular genetic characterization of animal genetic resources. FAO Animal Production and Health Guidelines. N ${ }^{\mathbf{0}}$. 9. Rome. $100 \mathrm{p}$.

Gómez, YM; Fernández, M; Rivera, D.; Gómez, G; Bernal, JE. 2013. Genetic characterization of colombian Brahman cattle using microsatellites markers. Russian Journal of Genetics 49(7):737-745.

Hernández, C; Oliveira, M; Ostos, H; Guerra, MT. 2009. Genetic variability of the Zebu cattle breed (Bos Indicus) in the departament of Huila, Colombia using Microsatellite Molecular Markers. Acta Biológica Colombiana 14(3):173-180.

Ichikawa, Y; Takagi, K; Tsumagari, S; Ishihama, K; Morita, M; Kanemaki, M; Takeshi, M. 2001. Canine parentage testing based on microsatellite polymorphisms. Journal of Veterinary Medical Science 63(11):1209-1213

INEC (Instituto Nacional de Estadística y Censo). 2015 VI Censo Nacional Agropecuario (en línea). 147 p. Consultado 1 ene. 2016. Disponible en http://www. mag.go.cr/bibliotecavirtual/a00338.pdf

ISAG (International Society for Animal Genetics). 2014 Xi'an, China. Cattle Molecular Markers and Parentage Testing Workshop (en línea). Consultado 1 ene. 2016. 7 p. Disponible en http://www.isag.us/ Docs/Workshop\%20report\%20CMMPT\%202014.pdf

Jamieson, A; Taylor, SC. 1997. Comparisons of three probability formulae for parentage exclusion. Animal Genetics 28(6):397-400.

Jones, AG; Small, CM; Paczolt, KA; Ratterman, NL. 2010. A practical guide to methods of parentage analysis. Molecular Ecology Resources 10(1):6-30.

Kalinowski, ST; Taper, ML; Marshall, TC. 2007. Revising how the computer program CERVUS accommodates genotyping error increases success in paternity assignment. Molecular Ecology 16(5):1099-1106.

Kios, D; van Marle-Koster, E; Visser, C. 2012. Application of DNA markers in parentage verification of Boran cattle in Kenya. Tropical Animal Health and Production 44(3):471-476.

Marshall, TC; Slate, J; Kruuk, LEB; Pemberton, JM. 1998. Statistical confidence for likelihood-based paternity inference in natural populations. Molecular Ecology 7(5):639-655. 
Martínez, M; Vargas, B; Cordero, JM; Chacón, I; León, B. 2015. Diversidad genética entre subpoblaciones raciales bovinas de Costa Rica. Agronomía Costarricense 39(2):33-46.

Řehout, V; Hradecká, E; Č́tek, J. 2006. Evaluation of parentage testing in the Czech population of Holstein cattle. Czech Journal of Animal Science 51(12):503509.

Riojas, VM; Gomez, JC; Garza, JM; Gallardo, DC; De Tellitu, JN; Wong, A; Davalos, G; Salinas, JA. 2009. Exclusion Probabilities of 8 DNA Microsatellites in 6 Cattle Breeds from Northeast Mexico. Journal of Animal and Veterinary Advances 8(1):62-66.

Stevanovic, J; Stanimirovic, Z; Dimitrijevic, V; Maletic, M. 2010. Evaluation of 11 microsatellite loci for their use in paternity testing in Yugoslav Pied cattle (YU Simmental cattle). Czech Journal of Animal Science 55(6):221-226.

Summers, K; Amos, W. 1997. Behavioral, ecological and molecular genetic analyses of reproductive strategies in the Amazonian dart-poison frog, Dendrobates ventrimaculatus. Behaviour Ecology 8(3):260-267.

Visscher, PM; Woolliams, JA; Smith, D; Williams, JL. 2002. Estimation of pedigree errors in the UK dairy population using microsatellite markers and the impact on selection. Journal of Dairy Science 85(9):2368-2375.

Waits, L; Luikart, G; Taberlet, P. 2001. Estimating the probability of identity among genotypes in natural populations cautions and guidelines. Molecular Ecology 10(1):249-256. 